\section{AB0555 ANTI-RO POSITIVITY EFFECT ON CLINICAL CHARACTERISTICS AND MORTALITY IN A SINGLE- CENTER COHORT OF ANTISYNTHETASE SYNDROME PATIENTS}

E. Bozzalla Cassione ${ }^{1}$, A. Biglia' ${ }^{1}$, G. Zanframundo ${ }^{1}$, S. Grignaschi ${ }^{1}$, L. De Stefano $^{1}$, P. Delvino ${ }^{1}$, E. Marasco ${ }^{1}$, C. Montecucco ${ }^{1}$, L. Cavagna ${ }^{1} .{ }^{1}$ University of Pavia, Rheumatology, Pavia, Italy

Background: Antisynthetase syndrome (ASSD) is an heterogeneous disease characterized by the occurrence of anti-synthetase antibodies (ARS) and the classic triad of arthritis, myositis and Interstitial Lung Disease (ILD). Pulmonary involvment bears an increased risk of life threatening events, being the main cause of mortality in these patients. No significant differences in mortality have been noticed among the different ARS specificities. However the role of extractable nuclear antigen antibodies on mortality in these patients is still matter of debate.

Objectives: To asses the effect of anti-RO antibodies on clinical characteristics and mortality in a large italian single-center cohort of ASSD patients.

Methods: Cohort study of outcome, clinical and laboratory characteristics, at baseline and at last follow-up, of 55 ASSD patients progressively enrolled and followed-up at our out-patient clinic. Survival was estimated with Kaplan-Meier function and comparison between frequencies of clinical characteristics was studied with Chi-square test.

Results: We identified 30 anti-Ro+ (M:F=1:4) and 25 anti-Ro- (M:F=1.5:1) ASSD patients. No difference in the age of onset was observed ( $p$ value $=0.83$ ). Classic triad prevalence and evolution was similar between the two groups with only significant difference of ILD prevalence at last follow up, higher in anti-Ro+ patients group ( $p$ value $=0.01$ ). Overall mortality was of 13 subjects $(23 \%)$ during the follow-up period (median 53 months, IQR 16-114). Despite the significantly higher pulmonary involvment in the anti-Ro+ group no difference between mortality in anti-Ro positive and negative groups was observed. Adjusting mortality to include disease-related events only did not provide any additional difference ( $p$ value $=0.5$ ) (Table 1). Survival curves of the two groups were not different at any time point (Log-rank test, p value 0.98) (Figure 1).

Table 1. Clinical and Demographic Characteristics of the cohort

\begin{tabular}{lccc}
\hline ASSD & Ro+ & Ro- & P value \\
\hline PZ (M:F) & $30(6: 24)$ & $25(14: 10)$ & $/$ \\
Age (years) at disease onset (median, IQR) & $56(43-69)$ & $54(46-68)$ & 0.83 \\
Disease duration (months) (median, IQR) & $55(23-112)$ & $52(13-130)$ & 0.681 \\
Arhtritis at onset (\%) & $22(73)$ & $17(68)$ & 0.664 \\
Arhtritis at last follow-up (\%) & $26(86)$ & $18(72)$ & 0.175 \\
Myositis at onset (\%) & $12(40)$ & $15(60)$ & 1 \\
Myositis at last follow-up (\%) & $19(63)$ & $21(84)$ & 0.086 \\
ILD at onset (\%) & $20(66)$ & $12(48)$ & 0.162 \\
ILD at last follow-up (\%) & $30(100)$ & $20(80)$ & 0.01 \\
Complete form at onset (\%) & $8(26)$ & $4(16)$ & 0.34 \\
Complete form at last follow-up (\%) & $17(56)$ & $13(52)$ & 0.729 \\
Raynaud phenomenon (\%) & $7(23)$ & $8(32)$ & 0.472 \\
Mechanic's hands (\%) & $12(40)$ & $12(48)$ & 0.551 \\
Death (\%) & $7(25)$ & $6(24)$ & 0.953 \\
Disease related death (\%) & $3(42)$ & $4(66)$ & 0.506 \\
\hline
\end{tabular}

\section{Survival curve}

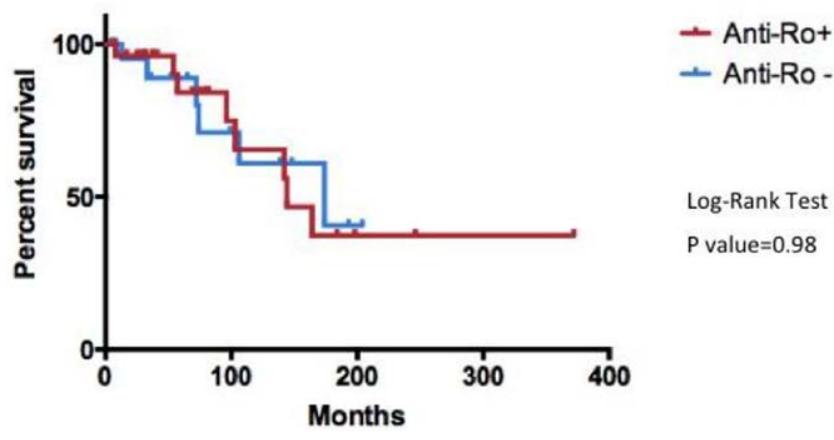

Figure 1. Kaplan-meier curve of anti-Ro+ and anti-Ro-ASSD patients.
Conclusion: The clinical spectrum and the time course of ASSD is not significantly affected by the presence of anti-Ro antibodies, althuough ILD seems to be more associated to anti-Ro antibodies. Despite higher prevalence of ILD in Ro positive group no difference in mortality was observed in respect to anti-Ro negative patients.

\section{References:}

[1] Cavagna L. Medicine (Baltimore) 2015 Aug;94(32):e1144

[2] Marie I. Semin Arthritis Rheum. 2012 Jun;41(6):890-9

Disclosure of Interests: None declared

DOI: 10.1136/annrheumdis-2020-eular.5615

\begin{tabular}{|l|l|}
\hline AB0556 & PRIMARY SYSTEMIC SCLEROSIS HEART \\
INVOLVEMENT (PSSCHI): A SYSTEMATIC \\
LITERATURE REVIEW (SLR), CONSENSUS- \\
BASED DEFINITION AND PRELIMINARY \\
VALIDATION.
\end{tabular}

C. Bruni ${ }^{1}$, M. H. Buch ${ }^{2}$, P. Seferovic ${ }^{3}$, M. Matucci-Cerinic ${ }^{1}$ on behalf of World Scleroderma Foundation and Heart Failure Association collaborators. ${ }^{1}$ University of Florence, Experimental and Clinical Medicine, Division of Rheumatology, Firenze, Italy; ${ }^{2}$ The University of Manchester, Rheumatology, Manchester, United Kingdom;

${ }^{3}$ University of Belgrade - Faculty of Medicine, Cardiology, Belgrade, Serbia

Background: pSScHI may cause tissue, functional and conduction abnormalities with varied clinical manifestations. The absence of a clear definition of pSScHI impairs the significance and ability of focussed research, frequently not allowing the distinction between primary and secondary involvement.

Objectives: We aimed to establish an expert consensus definition for pSScHI, to be used in clinical trials and everyday clinical practice, and to start its validation process.

Methods: A SLR for cardiac manifestations and alterations in SSc was conducted using PubMed, Web of Science and Embase. Articles published from inception to December $31^{\text {st }}, 2018$ were identified. Inclusion criteria included papers in English on adult SSc patients, with heart involvement as outcome. We excluded non-human studies, secondary heart involvement (eg PAH, drugs, infections), reviews and case reports. PRISMA recommendations were followed where applicable. Extracted data were categorized into relevant domains (signs, symptoms, anatomical site involved, physiological abnormalities, pathological changes, prognostic outcomes), which informed the consensus definition. Sixteen senior experts ( 7 rheumatologists, 8 cardiologists, 1 pathologist) discussed the data and, using a nominal group technique, added expert opinion, provided statements to consider and ranked them. Consensus was attained for agreement $>70 \%$. Sixteen clinical cases were evaluated in two rounds to test for face validity, feasibility, inter- and intra-rater reliability and criterion validity (gold standard set by agreed evaluation between expert rheumatologist, cardiologist and methodologist).

Results: 2593 publications were identified and screened, 251 full texts were evaluated, 172 met eligibility criteria. Data from the 7 domains were extracted and used to develop the World Scleroderma Foundation - Heart Failure Association (WSF-HFA) consensus-derived definition of pSSc-HI, as follows:

"pSScHI comprises cardiac abnormalities that are predominantly attributable to SSc rather than other causes and/or complications*. pSScHI may be sub-clinical and must be confirmed through diagnostic investigation. The pathogenesis of pSScHI comprises one or more of inflammation, fibrosis and vasculopathy. ${ }^{*}$ Non SSc-specific cardiac conditions (e.g. Ischaemic heart disease, arterial hypertension, drug toxicity, other cardiomyopathy, primary valvular disease) and/or SSc non cardiac conditions (e.g. PAH, Renal involvement, ILD)."

Face validity was determined by a $100 \%$ agreement on credibility; application was feasible, with a median $60(5-600)$ seconds taken per case; inter rater agreement was moderate [mKappa $(95 \% \mathrm{Cl}) 0.56(0.46-1.00)$ and 0.55 (0.44-1.00) for the two rounds] and intra rater agreement was good [mKappa $(95 \% \mathrm{Cl}) 0.77(0.47-1,00)]$. Content validity was reached based on the wide variety of patients in the SLR, criterion validity was reached with 78 (73-84) $\%$ correctness. 